\title{
$\longrightarrow$
}

\section{Subverting $\mathrm{T}_{\text {reg }}{ }^{-}$ mediated protection for tumors}

\section{By Kai-Jye Lou, Senior Writer}

$\mathrm{T}_{\text {reg }}$ cells present a conundrum for cancer researchers-although these cells can suppress the antitumor immune response, depleting them can trigger severe inflammation and autoimmunity. Now, a team of researchers at St. Jude Children's Research Hospital has uncoupled these effects by targeting neuropilin 1-semaphorin $4 \mathrm{~A}$ signaling, a pathway active in tumor-associated $\mathrm{T}_{\text {reg }}$ cells. ${ }^{1}$

The results suggest that disrupting the neuropilin 1 (NRP1)semaphorin 4A (SEMA4A) signaling axis could be used to treat tumors that respond poorly to immunotherapies designed to boost the antitumor immune response.

$\mathrm{T}_{\text {reg }}$ cells are mediators of peripheral tolerance and play a critical role in preventing pathological autoimmunity and inflammation. ${ }^{2}$ However, tumors are known to induce the expansion of $\mathrm{T}_{\text {reg }}$ cells to protect themselves from the host immune system. ${ }^{3}$ Indeed, multiple groups have shown that $\mathrm{T}_{\text {reg }}$ cells limit the efficacy of cancer immunotherapies. ${ }^{4,5}$

"We want to attack $\mathrm{T}_{\text {reg }}$ cells in cancer, but we know that broadly suppressing their activity or wiping them out could have devastating immunological consequences as patients with a mutation that renders them

"There are enough data here to be excited about potential therapeutic possibilities and to warrant additional investigation of this pathway, but we need to first determine how broadly it is utilized in various cancers and in other inflammatory states."

$$
\text { -Dario Vignali, }
$$
deficient in these cells have severe autoimmunity," said Dario Vignali, vice chair of the Department of Immunology at St. Jude Children's Research Hospital. "Thus, there is a strong therapeutic desire to manipulate $\mathrm{T}_{\text {reg }}$ cells in a manner that focuses the modulation on their activity in the tumor microenvironment."

Vignali's group thus searched for molecules and pathways that $\mathrm{T}_{\text {reg }}$ cells use in highly inflammatory settings such as the tumor microenvironment but not when maintaining immune system homeostasis.

The researchers used a series of transwell assay and transcriptome profiling studies to identify SEMA4A as a ligand that boosts $\mathrm{T}_{\text {reg }}$ cell function. A follow-up ELISA showed that SEMA4A interacted with
NRP1, a membrane-bound co-receptor expressed on $\mathrm{T}_{\text {reg }}$ cells whose function in the cells was previously unknown.

Mice with deletion of Nrp1 in $\mathrm{T}_{\text {reg }}$ cells did not develop autoimmunity, but the Nrp1-deleted $\mathrm{T}_{\text {reg }}$ cells still maintained immune system homeostasis.

In mice, $\mathrm{T}_{\text {reg }}$ cell-restricted deletion of $\mathrm{Nrp} 1$ resulted in a stronger antitumor immune response against injected cancer cells than no deletion. The Nrp1-deficient mice also showed less tumor growth and increased survival compared with nondeficient controls.

In wild-type mice injected with the cancer cells, SEMA4A- or NRP1targeted antibodies decreased tumor growth compared with control antibodies.

Subsequent gene expression analysis on the $\mathrm{T}_{\text {reg }}$ cells revealed an Nrp1-induced transcriptional signature associated with enhanced $\mathrm{T}_{\text {reg }}$ cell function and survival. Inhibition of Nrp1-signaling blocked this signature.

Results were published in Nature.

"This study suggests it could be possible to target this neuropilinsemaphorin pathway with antibodies or soluble antagonists to eliminate $\mathrm{T}_{\text {reg }}$ cells that suppress the antitumor immune response without perturbing the $\mathrm{T}_{\text {reg }}$ cells that keep autoimmunity in check," said Theresa Whiteside, a professor of pathology, immunology and otolaryngology at the University of Pittsburgh Cancer Institute.

Whiteside said that the study also presents a good model on how to search for pathways involved in the regulation of $\mathrm{T}_{\mathrm{reg}}$ function in tumors and inflammatory settings.

"We were able to use our data to create a set of analytical fingerprints to indicate when this pathway is active in $\mathrm{T}_{\text {reg }}$ cells, and this allowed us to show that the tumor microenvironment encourages $\mathrm{T}_{\text {reg }}$ cells to exploit this pathway, thereby evading the host immune system," added Vignali, who was the corresponding author. "We know from our study that removing NRP1 removes this fingerprint, which suggests there isn't a redundant pathway for this tumor-protective function in $\mathrm{T}_{\text {reg }}$ cells."

Vignali said that targeting the SEMA4A-NRP1 pathway in $\mathrm{T}_{\text {reg }}$ cells could provide a new approach to limit $\mathrm{T}_{\text {reg }}$ cell function in tumors and make tumor cells visible to the host immune system, which could be useful in cases in which cancer immunotherapies have shown limited efficacy.

\section{Determining applicability}

Vignali said that his group still needs to determine how broadly the SEMA4A-NRP1 signaling pathway in $\mathrm{T}_{\text {reg }}$ cells is exploited by various tumors.

"There are enough data here to be excited about potential therapeutic possibilities and to warrant additional investigation of this pathway, but we need to first determine how broadly it is utilized in various cancers and in other inflammatory states," he told SciBX.

Whiteside said that therapies based on targeting the SEMA4A-NRP1 pathway will likely need to be accompanied by a companion diagnostic to determine whether the pathway is active in a particular tumor.

She also thinks that there are likely to be other pathways that regulate the function of $\mathrm{T}_{\mathrm{reg}}$ cells in the tumor as well as subpopulations of 


\section{ANALYSIS}

$\mathrm{T}_{\text {reg }}$ cells that suppress the antitumor immune response via different mechanisms. Moreover, she said other cell populations such as myeloidderived suppressor cells are known to attenuate antitumor immunity and weaken the effect of immunotherapies. ${ }^{6}$

As a result, Whiteside thinks that targeting any one pathway may not be sufficient for controlling tumor-mediated immunosuppression.

Vignali said that the group now needs to run a more comprehensive set of studies to check for potential immunological consequences and side effects from targeting the SEMA4A-NRP1 pathway.

"Even though we show that inhibiting this pathway did not initiate an autoimmune response, we don't yet know if inhibiting this pathway will exacerbate a pre-existing autoimmune response, which may be present in patients," said Vignali. "Also, NRP1 is also expressed on neurons and is involved in their development, so we need to make sure blocking this pathway will not perturb these cells in an undesirable manner."

Whiteside wanted to see studies to determine whether the SEMA4ANRP1 pathway is active in metastatic tumors, which are the primary contributors to patient mortality in cancer and could have different characteristics than the primary tumor. Further along, she said, it will be important to determine whether compounds targeting the pathway will be sufficient on their own or need to be combined with other cancer therapies.

St. Jude Children's Research Hospital has filed for a patent covering the findings reported by Vignali's group. The work is available for licensing.

Lou, K.-J. SciBX 6(32); doi:10.1038/scibx.2013.849

Published online Aug. 22, 2013

\section{REFERENCES}

1. Delgoffe, G.M. et al. Nature; published online Aug. 4, 2013; doi:10.1038/nature12428

Contact: Dario A.A. Vignali, St. Jude Children's Research Hospital, Memphis, Tenn.

e-mail: vignali.lab@stjude.org

2. Vignali, D.A.A. et al. Nat. Rev. Immunol. 8, 523-532 (2008)

3. Kretschmer, K. et al. Immunol. Rev. 212, 163-169 (2006)

4. Curiel, T.J. J. Clin. Invest. 117, 1167-1174 (2007)

5. Colombo, M.P. \& Piconese, S. Nat. Rev. Cancer 7, 880-887 (2007)

6. Khaled, Y.S. et al. Immunol. Cell Biol.; published online June 25, 2013; doi:10.1038/icb.2013.29

COMPANIES AND INSTITUTIONS MENTIONED

St. Jude Children's Research Hospital, Memphis, Tenn. University of Pittsburgh Cancer Institute, Pittsburgh, Pa. 